\title{
THE LAW OF OCCLUSION AND THE NORMAL IN ORTHODONTIA*
}

\author{
A Philosophic Survey of Recent T'endfincies in Orthodontia in the \\ LIGHT OF SCIENCE
}

By A. LeRoy Johnson, D.M.D., Springfield, Mass.

$I^{\mathrm{N}}$ MODERN orthodontia there has developed a tendency to absolute methods and absolute claims as to the results of treatment. And it requires no violation of reason to see in such methods the logical extremes of the doctrines upon which orthodontia was founded. With its inception in mechanical dentistry during that period of philosophic thought when diversity of form was entirely attributed to environmental influences such conclusions as the following were ratural. They are the dominance of the mechanical influence of cusp relation in the development of occlusion; the inherent tendency of all organisms to evolve a definite type, harmonious in design, and the equal potentialities of development of all organisms. In the light of advancing science, and a deeper knowledge of the forces governing occlusion derived from practice we can no longer allow such doctrines to pass unquestioned even though they still express an element of truth.

The masticatory apparatus is a living part of a living thing and so is subject to the same general laws as are other parts of the organism. Hence the knowledge of its growth and development and the interpretation of such knowledge in terms of practical utility must conform to the methods and material of science in general.

The following is an attempt to express in terms of science the fundamental principle of orthodontia and the nature of the normal as it refers to the species and to the individual. In the subject matter is embodied a criticism of the doctrines referred to above.

The line between common knowledge and scientific knowledge is not easily drawn. To say that science is organized knowledge is not enough, for the commonest acts of everyday life are in greater or lesser degree organized. It can not be said that science is the more exact for much common knowledge is exact. Common knowledge may be just as precise as scientific knowledge but in order that it be scientific it must be precise. Science reduces other knowledge to the same degree of precision. From one point of view science may be called an extension of preception by means of reasoning. It is prevision. A child who knows that a lighted match thrust into water will go out, and the astronomer who, having calculated a transit of Venus, can delineate the black spot entering the sun's disc, do the same thing. Each has a preconceived impression of what will happen upon fulfilling certain requisite conditions. The difference is in the complexity of the process to see the result. ${ }^{1}$ In

* Read before the Eighteenth Annual Meeting of the American Society of Orthodontists, Chicago, Illinois, August 1, 2, 3, 1918. 
reality it is a difference in degree only. One is merely an extended and perfected form of the other. Science defined is knowledge which has been systematized and formulated with reference to the discovery of general truths or the operation of general laws. Science exists because in order to foresee the result of a complex process it is essential that the knowledge referring to this process be arranged in conventional form.

The material of science is accumulated as the result of observation, comparison and experimentation and the methods employed are referred to as either qualitative or quantitative. The prediction that a piece of lead is heavier than a piece of wood of equal size, is the qualitative method. It exhibits certainty but not completeness of foresight. On the other hand, the prediction that water will freeze at $32^{\circ} \mathrm{F}$. shows foreknowledge not only of the effect to be produced but of the magnitude at which the effect will be produced. This illustrates the qualitative and quantitative methods combined. The quantitative method applies a definite measure to phenomena as in the use of thermometers, scales, dynamometers and as in the modern methods of experimental analysis. Hence, only in proportion as knowledge becomes quantitative is it especially scientific. Only in the degree that it is measurable is it scientific in the highest sense and do its previsions become complete as well as certain.

From another aspect passing from qualitative to quantitative is passing from inductive science to deductive science. Inductive science is qualitative; it is reasoning from the individual to the universal. Induction gives general principles derived from the observation of individuals as in the formation of a natural law. Deductive science is quite the opposite from inductive science. It is reasoning from the general to the individual. Deduction gives explicit knowledge, it measures. Although deductive and quantitative methods are not coextensive all quantitative prevision is reached deductively. The first stage of all science is inductive but after continually applying this method with constant resu1ts, deductively the results of observation may be approached. The history of physiology and morphology shows many instances of the legitimate use of this method. The knowledge of the presence of the vascular and nervous system was inductively established and from this it has been possible to draw certain general deductions.

Thus do common knowledge, qualitative and quantitative science stand in the orderly scquence of their evolution. It is essential to grasp the significance of this sequence and also the distinction between qualitative and quantitative methods of scientific work. The quantitative work that has been done during the last quarter or possibly half of a century has upset many of the philosophical generalizations expressed even now in orthodontic literature.

From the definition of science it is clear that the selection and utilization of knowledge in each branch of science is determined by its relation to the general truth or law which gives to a particular science its distinctive character. In short all science is founded upon a general truth or law. In organic science the basic principle is referred to as a natural law.

A natural law or law of nature means nothing more than that there exists a uniformity in a certain phase of natural phenomena. It is the result of 
inductive or qualitative study, i. e., from the observation and comparison of many individual cases there is evident a certain uniformity of phenomena. In the words of Herbert Spencer, "A natural law is the uniformity seen in the diversity of organic forms." ${ }_{2}$

Now, orthodontia like all other branches of science is founded upon a general truth, a natural law. As a result of the study of many organisms, from the observation and comparison of the growth and development of the masticatory apparatus of the individuals of many species there is seen to be a certain uniformity of phenomena. From the viewpoint of inductive reasoning and as a result of qualitative study the order of sequence of the phenomena is seen to be so invariable under normal conditions that a uniformity is readily recognized. And since the mechanical relation of the occlusal inclined planes of the teeth, typical for the species, is the goal of the growth and development of this part of the organism, the uniformity, the natural law (manifest in the growth and development) we call the law of occlusion. Therefore, the law of occlusion is the general truth, expressing a uniformity seen in the diversity of organic forms, upon which the science of orthodontia is founded. It is the basic principle about which the knowledge of the growth and development of the masticatory apparatus, the subject matter of orthodontia is systematized and formulated into a science.

It is not an uncommon procedure in practice to confine the study of the orthodontic case to a consideration of the occlusal relations of the teeth. A concept called normal occlusion is the measure laid upon each individual case and as the occlusal relations depart from this normal occlusion the teeth are said to be in malocclusion requiring treatment. To arbitrarily determine the ideal relation of each individual inclined plane and then, by virtue of the plastic nature of bone, to mechanically conform all variations to this ideal relation, is, like many half truths, beautifully simple. And in this comparatively easy way the question is often disposed of.

The obvious unsoundness of such procedure is further emphasized in the diversity of opinion manifest in the literature as to the idea conveyed by normal occlusion. To some it is a concept derived from the study of dead forms, prehistoric skulls and lower species; a purely mental concept, a hypothesis, when applied to modern man. To others it is a condition of greatest occlusal contact for the individual case while a few consider normal occlusion as synomomous with natural occlusion. However, the latter do not confine their exposition to that which is typical of the species.

This confusion of ideas is due to a loose interpretation of terms and to the fact that studies of harmonic conditions of development have been limited almost entirely to organisms of a different nature or living under different conditions from the human organism of today. The conception of the normal has not been the result of evidential reasoning according to scientific standards.

What then is the meaning of the word normal? As the term is used in different branches of science slight variations in sense, appear, according to the nature of the material it is expressive of, and, also, as emphasis is put upon the words chosen to convey its meaning, as rule, standard, model, type 
or pattern. In chemistry normal denotes a solution of definite character; in geometry normal refers to any perpendicular line; in economics normal and natural are often used synonymously but the use of the word natural invokes certain metaphysical assumptions which are not connoted by the term normal; in biology normal is the balance of activity, growth and repair. Although the word is used differently in the different sciences, in each science its use conveys a definite idea. So in orthodontia, where the word normal plays such a vital role in terminology it is essential that it be used in as restricted a sense as possible.

In Webster we find that normal "is according to, constituting or not deviating from an established norm" and that a "norm" is a rule or authoritative standard. As used in the organic sciences normal is according to, constituting or not deviating from a standard.or rule which is observed or claimed to prevail in nature. The normal then is not synonymous with natural; it is a standard derived from a study of the natural.

Then if normal occlusion is to be a scientific guide in orthodontia, it must be a standard derived from a study of natural forms. Moreover it must be a standard derived from a study of natural forms as manifest in the human masticatory apparatus as it lives today in the 20th century. It is not a standard derived from the study of fossil forms, prehistoric skulls and lower species. A knowledge of such material is included in that larger concept the law of occlusion, and is necessary to an understanding of the phenomena encountered in the study of the human denture. But the material which determines the standard, the normal, which is to be taken as a tangible guide in diagnosis must be derived from the material we work upon.

The condition of the organized world is not a fixed condition; it is constantly changing. The man of today is not the man of yesterday. Changed conditions of living have caused some parts and organs to become less essential to existence. Until as Bateson says "We, animals, live not only by virtue of, but also in spite of what we are. Any instinct or organ may be of use; the real question is of how much use it is."3 In view of the diversity of form manifest in the human masticatory apparatus we may well question "of how much use it is." Do not misunderstand me. I do not infer that normal occlusion is not essential but that in the life history of the human organism and the present condition of its denture there is evidence of its not being as vital to existence as it was and is to those organisms from which the popular conception, the old orthodox ideal occlusion has been derived; the same conception that has been responsible for the belief in the dominance of the mechanical influence of cusp relation in the development of the dental arch. It seems quite obvious then that the conception of normal occlusion, typical of the species, as a scientific guide must be the result of the study of the masticatory apparatus of many human beings, living in states of health under the complex conditions of modern civilization. It is a standard derived from statistical investigation.

Today the most conservative thinkers do not question the natural tendency of all organisms to vary. The structural relations of one are not identi- 
cal with those of another. Hence in orthodontia we are forced to believe that the normal occlusion for the species, $i$ e., an average typical condition of $\dot{c}$ usp relation, determined by statistical investigation, and the normal of the individual are not necessarily the same. In the majority of cases we may reasonably expect to find a natural difference between the species normal and the normal of the individual. And thus we are led to one of the most momentous questions of orthodontia, to determine the normal of the individual, to ascertain the range of variation from the normal of the species which is consistent with the most complete realization of the growth and developmental possibilities of the individual organism as a whole. A definite solution of this problem is not anticipated in any instance, in fact the absolute claim is unwarranted. But because now it seems hopelessly beyond our reach is no reason why we should not face the facts of science, utilize its available material and conform our efforts to its methods.

To determine the normal of the individual is a difficult problem. It involves the consideration of phenomena fundamental in organic life. And a scheme of investigation or system of diagnosis which will indicate the individual normal and so in a degree at least anticipate the possible and the impossible in treatment, must be built upon a knowledge of normal and pathological processes as they are expressed in growth and development. We can not postulate the role of pathology as an etiologic factor of malocclusion until we are familiar with normal processes. These latter include such fundamental problems of biology as the interdependence of function and structure; variation and adaptation; heredity and the unity of the organism as a whole. In the material of science relating to these so-called normal processes the individual element in the organism is clearly emphasized.

From the viewpoint of philosophy the life of the individual organism is a continual balancing of external by internal forces. Fisk says, "that it is the continuous adjustment of internal to external. conditions." presses the same thought in different terms. Thus each consider two kinds of equilibration briefly designated as internal and external. Fisk referring to the internal processes says "we see that direct equilibration which consists in continually arranging all the units of the organism in accordance with their physiologic polarities, exemplified alike in heredity and in the correlation of growth. The dwindling and final evanescence of organs which are disused is due to the fact that the nutritive material is all needed by the other organs which are in constant use." Here Fisk foreshadowed in philosophy the research work of recent years in the necessity of considering the organism as a whole in the endeavor to determine the normality of any of its parts. Philosophically considered a normal individual organism is one expressing an adjustment of internal to external forces consistent with the most complete growth and development of the organism, as a whole. Certain organs develop and atrophy to maintain this equilibrium.

In studying the question of the individual normal it is of particular interest to note the change in the attitude of science to the problem of adaptation, and to follow the investigation in physiologic chemistry and heredity. 
For several decades after Darwin the literature was filled with philosophical speculations relative to the phenomena of adaptation. Students of evolution found the study of it so much more interesting and impressive than that of specific diversity that they seem to have seen nothing else. However, during this period very little progress was made. And it was not until the introduction of experimentation on the basis of the quantitative method by the school of experimental biologists headed by Jacques Loeb that definite progress was made.

Approached from a new angle the problem of adaptation appears in a very different light. Loeb says "that authors have too often spoken of adaptation to environment where environment was not responsible for the phenomena." And "while it is possible for forms with moderate disharmonies to survive, those with gross disharmonies can not exist and we are not reminded of their possibie existence. As a consequence the cases of apparent adaptation prevail in nature." In another place he says, "Those organisms live and develop that are free from the grosser type of disharmonies; the rest are doomed on account of a gross lack of harmony of the parts. Those latter we never see and this gives us the erroneous conception that harmony or design is the general character of living matter." In his work "The Organism As a Whole" the evidence and references are clearly set forth from which the conclusions as to the lack of design in living nature are derived.

In the work upon the internal secretions physiologic chemistry is applying direct and penetrating methods to biological problems. Mathews says:

"The body is an organic whole, and these so-called organs of internal secretion are not unique, but the bones, muscles, skin, brain, and every part of the body are furnishing internal secretions necessary to the development. and proper functioning of all the other organs of the body. Such a scheme to be complete must embrace every organ; only the barest beginning has been made in this study, so important, so necessary for the understanding of development and inheritance. The problems of development and inheritance can not be solved until these physiologic questions are answered."

It is claimed to have been demonstrated that what has appeared as adaptation when unknown has turned out to be a result of the action of a definite chemical substance circulating in the blood. Leo Loeb observed that "the corpus luteum of the ovary gives off a substance to the blood which alters the tissues in the uterus in such a way that contact with any foreign body induces the same decidua formation as does the egg." Steinach has shown that "the wonderful mating instincts seem to be due to definite substances secreted by the sex glands." I,ewis has shown "that if the optic cup is transplanted under the skin of a young larva into any part of the body the skin in contact with the optic cup will form a lens; it looks as if a chemical substance from the optic cup were responsible for the formation of the lens." ${ }^{10}$

In the work of the mutationists and students of Mendelian heredity there is evidence that disharmonic conditions of development may express alterations in the hereditary constitution. In the Scientific Monthly, May, 1918, Morgan says :

"A specific gene may be essential to the normal development of a certain 
organ, which organ through an internal secretion may affect other parts of the body, or even the body 'as a whole.' If, for example, the development of the thyroid gland were known to be dependent on the presence of a certain gene (amongst all of the others involved in its formation) a change in the postulated gene leading to the arrest in the development of the thyroid gland would, owing to the lack of a sufficient amount of some internal secretion of that gland, produce a malformed child with all of the various stigmata of the cretin."

If this is true then the factors of heredity can not be eliminated even though the phenomena directly responsible for the development of a deformity can be traced to the abnormal activity of a ductless gland.

It is neither necessary nor wise to accept in detail the conclusions of any one man or class of men in an attempt to establish a scientific truth In experimental biology it is never certain that all conditions are constant except the one being studied. Consequently it is quite a common circumstance for two investigators of ability to arrive at conclusions diametrically opposed. Furthermore, it is always difficult to determine the value of evidence and particularly so when the alleged phenomena are of exceptional significance. The criterion of science in its three phases, observation, comparison, and experimentation is not always applicable in the study of the life phenomena of the human organism. Hence, the best that we can do in many instances is to apply the "test of inconceivability" to the evidence presented; believing things when we must, but not before.

However, from the most conservative standpoint of accepted scientific thought the doctrines proclaimed in the early days of modern orthodontia are now known to be inadequate. The evidence of science is explicit that the nasticatory apparatus is a dependent and living part of a living organism; that it is controlled in its growth and development by the same factors which control the growth and development of the organism as a whole, and that it is expressive of both physical and psychical phenomena. We can no longer assume that all individuals possess equal possibilities of development. "Studies in modern genetics are emphasizing the immense, the overwhelming importance of heredity, in both phylogeny and ontogeny." (Conklin. ${ }^{11}$ ) And it must be remembered that the directing and guiding factors of development are present in the organization of the germ cell, while environmental factors exercise a stimulating, inhibiting and modifying influence on development. Heredity fixes the possibilities of development; it sets bounds beyond which we can not pass. Nor can we assume the inherent tendency of all organisms to evolve a definite type, harmonious in design, in the face of evidence of natural disharmonic development and the possibility expressed upon good authority that such disharmonies may in rare instances be of the character of hereditary mutations.

The relative importance of heredity and environment is not the same in the development of man as in the development of the lower animals; environment plays a larger influence because of the longer period of development while heredity plays the same part as in the lower animals. Although most deformities are considered as the result of extrinsic, environmental forces the nature of the condition can not be known without taking heredity as well as environment 
inito consideration because the character of a response to stimuli is determined by the organism. The forms which structures take on are not the result of either environment or heredity working independently. Conklin says, "We do not inherit disease but the factor which determines whether one organ is susceptible to a disease while another is not, may be an inherited factor." ${ }^{2}$ Form development is the result of the interaction of forces determined by heredity and environment. Hence the etiologic factor of malocclusion is that which affects the organization of the organism or modifies the stimuli of the environment sufficiently to disturb the equilibrium expressed in normal development. A certain mechanical influence which will bring about a modification in the structural formation of the dental arch of one individual will not necessarily bring about the same arch formation in another individual. In each case we are face to face with the individual element.

'The problem of orthodontia is a complex one. Founded upon the law of occlusion and concerned with the development of the dental arch of the individual organism it involves the most fundamental problems of biology. A conception of orthodontia limited to the mechanical relations of tooth forms, while alluring in its simplicity and the promise of the absolute, will not withstand the crucial test of practical utility, since it ignores the essential vital activity of life phenomena.

\section{BIBLIOGRAPHY}

1, 2Spencer, Herbert: Essays-Scientific, Political and Speculative.

${ }^{3}$ Bateson, William: Materials for the Study of Variation.

+Fisk, John: Cosmic Philosophy.

5. $6,7,9,10$ Loeb, Jacques: The Organism as a Whole.

sMathews, Albert P.: Physiological Chemistry.

11. 12Conklin, Edwin Grant: Heredity and Environment in the Development of Man. 